\title{
DARBOUX'S PRINCIPLES OF GEOMETRY.
}

\section{Principes de Géométrie Analytique. By Gaston Darboux.} Paris, Gauthier-Villars, 1917.

A MONTH before his death Darboux wrote the preface to this, his last treatise. It presents in coordinated form lectures which he had delivered at intervals since 1872 either at the Sorbonne or the Ecole Normale. In the preface he announces that "the essential object of the book is the precise development of the notions relative to the imaginary and to infinity, and to show that in geometry they have the same place and importance as has been attributed to them in analysis for a long time." This idea is one which had very strong hold on Darboux's mind during his whole life. We find evidences of it in his first treatise, Sur une Classe remarquable de Courbes et de Surfaces algébriques et sur la Théorie des Imaginaires, published in 1872 , which deals largely with material presented in better form in part of the present volume. He touched upon the idea in his address before the Fourth International Congress at Rome in 1908. Moreover, the results of his own researches, in which isotropic elements are so effectively used, bear fitting testimony to the force of his contention.

The treatise consists of five books, or parts. The first has an introductory chapter dealing briefly with the history of the development of the ideas of infinity and imaginary elements, and an indication of the value of these entities and of homogeneous coordinates. Darboux remarks that the moderns differ from the ancients in their aim to reduce geometrical problems to general principles. His own efforts along this line in the field of differential geometry were rewarded with results which constitute one of his chief glories. The next three chapters deal respectively with tetrahedral coordinates, homographic point transformations, anharmonic ratio, abridged notation of Bobillier and plane homologies. The treatment is essentially the same as is to be found in other developments of these ideas from the analytic point of view. It is necessary to treat these ideas fully, since the treatise is intended for students who have had merely an introductory course in cartesian analytical geometry of two dimensions. Similar characterization may be given the next three chapters, 
which are short, and which deal with biaxial homologies, the principle of duality and correlation respectively. The last chapter of the first book deals with the well known projective properties of conics treated analytically.

In the second book isotropic elements are defined analytically and used in the development and refinement of the conceptions of distance and angle. The first chapter deals with metrical relations in the plane. The formula of Laguerre for the angle $\alpha$ between two lines $D$ and $D^{\prime}$, namely, that $e^{2 i a}$ is equal to the cross ratio of the lines $D, D^{\prime}$ and the isotropic directions of the plane, is derived, and applied to the derivation of the formulas of the trigonometry of triangles, whether real or imaginary. The isotropic lines through two points $A, B$ in a plane meet in two other finite points $A^{\prime}, B^{\prime}$ called by Darboux associate to the first. If $M$ is any other point of the plane, we have

$$
\frac{A M}{B M}=(-1)^{k} e^{i \widehat{B^{\prime} M A^{\prime}}}
$$

$k$ being an integer. By means of this formula Darboux shows that in a double infinity of ways the circle is the locus of a point whose distances from two fixed points, or poles, are in constant ratio. Generalizing this result, he studies during the remainder of the chapter the curves satisfying the condition $R_{1} R_{2} \ldots$ $R_{n}=k r_{1} r_{2} \cdots r_{p}$, where $k$ is constant, $R_{i}$ is the distance of any point of the curve to a fixed point $A_{i}$ and $r_{j}$ the distance to a point $a_{j}$.

In chapter two the methods and ideas previously developed are applied to the determination and study of two classes of curves, obtained as solutions of the two problems:

To find the curve whose tangent at any point intercepts on $n$ fixed lines of the plane segments which, measured from any fixed points on the lines, are in the most general linear relation.

To find the curve enveloped by a line which forms with $n$ couples of fixed lines $n$ triangles whose perimeters are in linear relation.

The imaginary circle at infinity, the isotropic plane, and null sphere, or isotropic cone, are defined analytically in chapter three; the idea of distance is made precise, and the angle between two lines is expressed in terms of cross ratio.

One of the best examples of the simplicity introduced into analytical geometry by the use of isotropic elements is fur- 
nished by taking the rectilinear generators of the sphere for its parametric lines. These parameters are introduced in the fourth chapter, and the relation between rotations of the sphere and the linear fractional transformation is established. Having proved that the cross ratio of any four points on a circle of the sphere is equal to the cross ratio of the four generators of the one system through these points, and also of the four generators of the second system through the points, Darboux establishes the following fundamental formula

$$
\frac{\tan \frac{1}{2} A M}{\tan \frac{1}{2} A M^{\prime}}=e^{i \overline{\gamma \gamma^{\prime}}},
$$

where $A M$ and $A M^{\prime}$ are two arcs of great circles, and $\gamma$ and $\gamma^{\prime}$ are poles of these circles, suitably chosen so that conventions as to signs shall be maintained. This formula forms the basis for the derivation, in the next chapter, of the formulas for the trigonometry of the sphere, such that the sides of the triangle are not required to be real, nor in the real case restricted in magnitude.

If $A$ and $B$ are two points of the sphere not on the same generator, the two generators through $A$ meet the two generators through $B$ in two points $A^{\prime}$ and $B^{\prime}$ other than points on the circle at infinity. The segments $A B$ and $A^{\prime} B^{\prime}$, which evidently are polar to one another with respect to the sphere, are called associate segments. If $a$ and $b$ are points diametrically opposite to $A$ and $B$, the four generators of the first system $d, d_{1}, d_{2}, d_{3}$ through these points meet the four generators of the second system $\delta, \delta_{1}, \delta_{2}, \delta_{3}$ in twelve points at finite distance. We indicate them as follows:

$$
\begin{array}{cccccccccccc}
A & B & A^{\prime} & B^{\prime} & A^{\prime \prime} & B^{\prime \prime} & a & b & a^{\prime} & b^{\prime} & a^{\prime \prime} & b^{\prime \prime} \\
d \delta_{1} & d_{2} \delta_{3} & d_{2} \delta_{1} & d \delta_{3} & d \delta_{2} & d_{1} \delta_{3} & d_{1} \delta & d_{3} \delta_{2} & d_{1} \delta_{2} & d_{3} \delta & d_{2} \delta & d_{3} \delta_{1} .
\end{array}
$$

In the last chapter of the second book, Darboux makes use of this enlarged system of points to study analytically the geometry on the sphere. He establishes the following fundamental system of formulas in which $\gamma_{A}$ denotes the distance of any point $M$ on the sphere to the point $A$, and $S_{A B M}$ the area of the triangle $A B M$ :

$$
\frac{\gamma_{A} \gamma_{B}}{\gamma_{A^{\prime}} \gamma_{B^{\prime}}}=\frac{\gamma_{a} \gamma_{b}}{\gamma_{a^{\prime}} \gamma_{b^{\prime}}}=\cos \frac{A B}{2}, \quad e^{i \widehat{A M B}}=\frac{\gamma_{A^{\prime}} \gamma_{b^{\prime}}}{\gamma_{B^{\prime}} \gamma_{a^{\prime}}},
$$




$$
\begin{aligned}
& e^{i / 2} S_{A M B}=\frac{\gamma_{b^{\prime}}}{\gamma_{a^{\prime}}}, \quad e^{i / 2} S_{a^{\prime} b^{\prime} M}=\frac{\gamma_{A}}{\gamma_{B^{\prime}}}, \\
& e^{i(\widehat{B A M}+\widehat{M B A})}=\frac{\gamma_{B^{\prime}} \gamma_{b^{\prime}}}{\gamma_{A^{\prime}} \gamma_{a^{\prime}}}=\frac{\sin B^{\prime} M}{\sin A^{\prime} M} .
\end{aligned}
$$

These equations are applied to the determination and study of certain curves on the sphere analogous to those for the plane previously referred to.

Book Three is devoted to the proof of the celebrated theorems of Poncelet concerning polygons inscribed in one conic and circumscribed to a second conic. The analysis is rendered simple and elegant by the use of a coordinate system in the plane suggested by the Chasles representation of a quadric on a double plane. Each point of the plane is determined by the intersection of two tangents to a basal conic $K$. By a convenient choice of the equation of $K$ the equations involved are given a simple form. After developing this coordinate system, the following theorems of consequence in the subsequent study are established:

$A$. If $n$ lines are defined by equations of the form $A_{i}=0$ $(i=1, \cdots, n)$, the general equation of curves of order $n-1$ passing through all the common points of the lines is

$$
\frac{a_{1}}{A_{1}}+\cdots+\frac{a_{n}}{A_{n}}=0,
$$

the constants $a_{i}$ being arbitrary.

$B$. If a curve of order $n-1$ contains all the points of intersection of $n$ tangents to a conic, it contains also the intersections of an infinity of other systems of $n$ tangents to the same conic, each system being determined by a value of a certain parameter.

These theorems are applied at the beginning of the second chapter to the proof of Poncelet's theorem: When a polygon is circumscribed to a conic $K$ and inscribed in a conic $C$, there exists a continuous suite of polygons of the same number of sides satisfying the same conditions. Moreover, in each case the points of intersection of any two sides of the polygon describe a conic or a straight line. After obtaining further properties of Poncelet polygons, Darboux gives an analytical explanation of the impossibility of obtaining such polygons when the conics $C$ and $K$ are chosen arbitrarily. 
When a polygon of $n$ sides is circumscribed to a conic $K$ and $n-1$ of its vertices, in a displacement, describe conics in the same tangential pencil* with $K$, the other vertex describes a conic of the pencil. This general theorem of Poncelet is the subject of the last chapter of the third book. Darboux gives a geometrical proof of the case $n=3$, and then extends the proof to the general case. Afterwards an analytical proof of the theorem is given. The chapter closes with a discussion of the forms given by Chasles to this theorem.

In his early writings Darboux made use of the ideas of distance and angle which grew out of Cayley's work. In 1895 he developed in a course of lectures at the Sorbonne the theory of Cayleyan geometry. In revised form these lectures constitute Book Four of the present treatise. In the first chapter we find an analysis of the origin of Cayleyan geometry. There is a full geometrical and analytical discussion of the absolute and of the consequences of its use.

Displacements in Cayleyan space are those homographic transformations which leave invariant the absolute, and thus preserve distances and angles. Darboux shows, in the second chapter, that in order to obtain a continuous transformation of this kind it is necessary and sufficient to subject the parameters of the two families of rectilinear generators of the absolute to any two linear substitutions.

When one of these substitutions is the identity, in the corresponding displacement all points of space are displaced the same distance. For the general case, in every displacement there are two lines, $D$ and $\Delta$, polar with respect to the absolute, which slide over themselves; they are called the axes of the displacement and are real for a real displacement. These displacements are of three types, all of which may be real: helicoidal, when four points only of the absolute remain fixed; rotations, when all the points of either axis remain fixed; half-turns (retournements), when all the points of both axes are fixed. These results are derived geometrically, and then the equations of the displacements of various kinds are obtained in simple form, when the equation of the absolute is $x y+z t=0$.

In the first part of chapter three the formulas of Cayleyan trigonometry are derived. Then follow expressions for volume and area. A sphere being defined as the locus of points equi-

* All the conics tangent to four lines form a tangential pencil. 
distant from a fixed point, two cases present themselves according as the latter point does or does not lie on the absolute.

The greater part of this book deals with finite elements, but occasionally there are sections dealing with differential geometry. The exposition throughout is so clear and simple that a college student might very well use this treatise as an introduction to this field of study.

The last book deals with inversion and with geometric concepts in whose field this transformation plays an important rôle. As a student in Paris, Darboux became interested in this subject and his first papers dealt with curves and surfaces for which these transformations are of peculiar significance. Out of them grew his remarkable thesis, Sur les surfaces orthogonales, and his first treatise previously referred to. In these publications are to be found the essential elements of the present book, so that here we have Darboux renewing his youth and embellishing its products with the style and finish acquired through the years.

Darboux begins with the following geometrical definition of inversion: Consider a sphere $S$, called the principal sphere of the inversion; the transform of a point $M$ is the unique point $M^{\prime}$ such that the null spheres with centers at $M$ and $M^{\prime}$ meet $S$ in the same circle. From this he derives the well known equations of inversion. The first chapter is devoted to the determination of the essential properties of inversion. It should be noted that these are obtained by geometrical reasoning and not by analytical processes. In particular, isotropic lines are transformed into isotropic lines. Here is introduced the isotropic developable, that is, the developable whose generators are isotropic lines. These surfaces, which are transformed into surfaces of the same kind by inversion, play an important rôle in many of Darboux's writings. In one of his first papers he called attention to the fact that an isotropic developable circumscribing a surface meets it along a line of curvature. A double curve on the isotropic developable circumscribing a surface is the locus of null spheres doubly tangent to the surface. These are called the focal curves of the surface, and each point is a focus of the surface. This definition is a generalization of Plücker's for foci of a plane curve and is in accord with Chasles's definition of focals for quadrics. 
If we have five fixed spheres such that any two meet one another orthogonally, and $s_{i}$ denotes the power of a point with respect to the sphere $S_{i}$ and $r_{i}$ its radius, the following identities hold for any point:

$$
\sum_{i=1}^{5}\left(\frac{s_{i}}{r_{\iota}}\right)^{2}=0, \quad \sum_{i=1}^{5} \frac{s_{\imath}}{r_{i}{ }^{2}}=-2, \quad \sum_{i=1}^{5} \frac{1}{r_{\iota}{ }^{2}}=0 .
$$

When one of the spheres $S_{i}$ is a plane, these identities hold provided that in the first the term $s_{i} / r_{i}$ be replaced by twice the distance from the point to the plane, and in the last two identities the corresponding term be omitted. The quantities $x_{i}$ defined by

$$
x_{i}=\lambda \frac{s_{i}}{r_{i}},
$$

where $\lambda$ is a factor of proportionality, are called the pentaspherical coordinates of the point. From the first of (1) we see that they must satisfy the condition

$$
\sum_{i=1}^{5} x_{i}{ }^{2}=0 \text {. }
$$

Moreover, the factor $\lambda$ is determined in accordance with the second of (1) by

$$
\sum \frac{x_{i}}{r_{i}}=-2 \lambda .
$$

This coordinate system is introduced in the second chapter and various properties are obtained, including the relations between the pentaspherical and rectangular coordinates of a point. In Darboux's opinion these coordinates bear a peculiar relation to inversion just as homogeneous point coordinates do to homographic transformations and tangential coordinates to correlations. The contents of this chapter are in the main equivalent to those set forth by Darboux in the first volume of his Leçons sur la Théorie générale des Surfaces with the difference that in the present treatise there are fewer applications to differential geometry. In the present treatment these coordinates appear in their historical setting. For Darboux was lead to invent this system as a result of his study of cyclides, i. e., the surfaces of the fourth degree with the circle at infinity as a double curve and the surfaces of the third degree passing through this circle. The remainder of the treatise deals with the study of these surfaces. 
According to Moutard, a surface is anallagmatic when it is transformable into itself by inversion. He showed that the envelope of the spheres orthogonal to a fixed sphere $S$ possesses this property when $S$ is the principal sphere of the inversion and that all anallagmatic surfaces can be obtained in this way. He called the center of $S$ the principal pole of the surface and the locus of the centers of the spheres the directrix surface. He announced the following properties of the cyclides:

Every cyclide is anallagmatic in five different ways; the line joining any two principal poles is perpendicular to the plane of the other three; any two of the five principal spheres cut orthogonally; the directrix surfaces are homofocal quadrics, which are central quadrics when the surface is of the fourth order and paraboloids when of the third order; each sphere doubly tangent to the surface meets it in two circles; each principal pole of a cyclide of the fourth order is the vertex of a quadric cone whose generators are doubly tangent to the surface, the curve of contact being on a sphere concentric with the directrix quadrics, and through each point of the surface pass in general ten circular sections.

These results are established by Darboux in the third chapter by analytical processes. The equation in cartesian coordinates of the general cyclide is of the form

$$
\varphi_{0}\left(x^{2}+y^{2}+z^{2}\right)^{2}+2 \varphi_{1}\left(x^{2}+y^{2}+z^{2}\right)+\varphi_{2}=0,
$$

where $\varphi_{0}, \varphi_{1}, \varphi_{2}$ denote functions of $x, y$ and $z$ of the degrees indicated by the subscripts. Darboux gives these functions normal forms before proceeding to the proof of the above result. $\mathrm{He}$ establishes also the existence of quadrics $V$ inscribed in the cyclide of fourth order along curves lying on concentric spheres; the five cones referred to above are quadrics. Since a cyclide of the third order can be regarded in an infinity of ways as a homographic transform of a general cubic surface, certain properties of the latter are studied in relation to the generalized inversion proposed by Hirst.

We remarked that Darboux was led to pentaspherical coordinates by his study of the cyclides. When the five fixed spheres referred to in Moutard's results are taken for the coordinate spheres of a pentaspherical system, the equation of the general cyclide is of the form

$$
\sum_{i=1}^{5} a_{i} x_{i}=0,
$$


as Darboux shows in chapter four. In this chapter and the next he derives from this analytical point of view the properties of the cyclides mentioned above, and many others.

Darboux's first important discovery was the existence of triply orthogonal systems of homofocal cyclides which constitute the most direct generalization of the well known triple system of confocal quadrics. The equation of such a system in pentaspherical coordinates is

$$
\sum_{i=1}^{5} \frac{x_{i}^{2}}{a_{i}-\lambda}=0,
$$

where the $a$ 's are constants, and $\lambda$ is a parameter, such that through each point of space pass three cyclides corresponding to three values of $\lambda$. Chapter six is devoted to a study of these systems.

The final chapter of the treatise deals with a point transformation first made known by the author in 1865. This transformation is defined analytically by the equations

$$
\frac{X}{x}=\frac{Y}{y}=\frac{Z}{z}=\frac{2 a}{x^{2}+y^{2}+z^{2}+a},
$$

where $a$ is a positive or negative constant. A combination of this transformation and the general homographic one gives the most general transformation which makes a plane correspond to a sphere. To every point $M(X, Y, Z)$ there correspond two points $m$ and $m^{\prime}$ of coordinates $x, y, z$ and $x^{\prime}, y^{\prime}, z^{\prime}$, which are the centers of null spheres meeting the sphere $S$, defined by

$$
x^{2}+y^{2}+z^{2}-a=0,
$$

in the circle of intersection of $S$ and the polar plane of $M$ with respect to $S$. We will not enumerate the various results obtained by Darboux, but will content ourselves with his observation that this transformation permits one to substitute for Cayleyan geometry a new geometry in which circles orthogonal to a sphere play the rôle of straight lines.

The reader of this treatise is sure to be impressed by the striking use which Darboux has made of isotropic elements. By the use of them and of frequent geometrical reasoning he has been enabled to avoid tedious stretches of analytical work. It is the simplicity and elegance of the processes which impress one. 
It seems almost superfluous to refer to the style of presentation of the results in this treatise. Every one in the least familiar with Darboux's writings is conscious of their lucidity and charm of style. And here we have the final product of this great expositor.

L. P. Eisenhart.

\section{SHORTER NOTICES.}

On Growth and Form. By D'Arcy W. Thompson. Cambridge, University Press, 1917. 8vo. 16+793 pages. Price 21 shillings.

UNTIL his recent appointment to the chair of natural history at the University of St. Andrews, the author of this work was professor of natural history at University College, Dundee. He has long been prominent as a member of various fishery boards and conferences, serving, for example, as the British delegate to the Bering Sea fishery conference of 1897. He is the author of "A Glossary of Greek Birds," has edited Aristotle's Historia Animalium and various blue-books on North Sea investigations, and he has translated and edited Hermann Müller's Fertility of Flowers.

And now we have this elaborate volume containing the results of many years of observation and study in widely separated fields and constituting an "easy introduction to the study of organic form." The author tells us: "It is not the biologist with an inkling of mathematics, but the skilled and learned mathematician who must ultimately deal with such problems as are merely sketched and adumbrated here. I pretend to no mathematical skill, but I have made what use I could of what tools I had; I have dealt with simple cases, and the mathematical methods which I have introduced are of the easiest and simplest kind."

After the Introduction we have chapters with the following titles: 2. On magnitude; 3 . The rate of growth (106 pages); 4. On the internal form and structure of the cell; 5 . The forms of cells; 6 . A note on absorption; 7-8. The form of tissues or cell-aggregates; 9. On concretions, spicules, and spicular skeletons; 10. A parenthetic note on geodetics; 11. The 